\title{
Numerical Analysis of Second-Order Mean Wave Forces by A Stabilized Higher-Order Boundary Element Method
}

\author{
Shao, Yanlin
}

Published in:

Journal of Offshore Mechanics and Arctic Engineering

Link to article, DOI:

$10.1115 / 1.4042197$

Publication date:

2019

Document Version

Peer reviewed version

Link back to DTU Orbit

Citation (APA):

Shao, Y. (2019). Numerical Analysis of Second-Order Mean Wave Forces by A Stabilized Higher-Order Boundary Element Method. Journal of Offshore Mechanics and Arctic Engineering, 141(5), [051801]. https://doi.org/10.1115/1.4042197

\section{General rights}

Copyright and moral rights for the publications made accessible in the public portal are retained by the authors and/or other copyright owners and it is a condition of accessing publications that users recognise and abide by the legal requirements associated with these rights.

- Users may download and print one copy of any publication from the public portal for the purpose of private study or research.

- You may not further distribute the material or use it for any profit-making activity or commercial gain

- You may freely distribute the URL identifying the publication in the public portal 


\title{
Numerical Analysis of Second-Order Mean Wave Forces by A Stabilized Higher Order Boundary Element Method
}

\author{
Yan-Lin Shao ${ }^{\mathrm{a}^{*}}$ \\ ${ }^{a}$ Department of Mechanical Engineering, Technical University of Denmark, 2800 Kgs. Lyngby, Denmark
}

\begin{abstract}
A stabilized Higher-Order Boundary Element Method (HOBEM) based on cubic shape functions is presented to solve the linear wave-structure interaction with the presence of steady or slowly varying velocities. The $m$-terms which involve second derivatives of local steady flow are difficult to calculate accurately on structure surfaces with large curvatures. They are also not integrable at the sharp corners. A formulation of the Boundary Value Problem (BVP) in a body-fixed coordinate system is thus adopted, which avoids the calculation of the $m$-terms. The use of body-fixed coordinate system also avoids the inconsistency in the traditional perturbation method when the second order slowly varying motions are larger than the first order motions.

A stabilized numerical method based on streamline integration and biased differencing scheme along the streamlines will be presented in this paper. The presence of convective terms in the kinematic and dynamic free surface conditions will lead to unstable solutions if the explicit method is used. Thus, an implicit scheme is used in this paper for the time integration of kinematic and dynamic free surface conditions. In an implicit scheme, solution of an additional matrix equation is normally required because the convective terms are discretized by using the variables at current time step rather than that from the previous time steps. A novel method that avoids solving such matrix equation is presented in this paper, which reduces the computational efforts significantly in the implicit method. The methodology is applicable on both structured and unstructured meshes. It can also be used in general second order wave-structure interaction analysis with the presence of steady or slowly varying velocities.
\end{abstract}

\section{INTRODUCTION}

Several rigs and FPSOs in the Norwegian and UK offshore sectors have experienced mooring system failures in recent years. Most of these incidents have occurred in moderate-to-heavy sea states, typically estimated to be 1-year storms. A direct cause of these failures is believed to be the overloading of the mooring system in extreme and steep waves, or wave groups that lead to larger slowly varying wave drift forces that result in larger offsets than that predicted by standard 
prediction tools. This perhaps has combined with other unfavorable circumstances like trenching of mooring lines. A comprehensive review of the start-of-the-art numerical modeling of wave-drift forces and motions is presented in Nestegård \& Shao (2014).

An accurate estimation of the wave drift forces in the presence of current is of concern when dealing with such failures. Stansberg et al. (2015) identified from earlier experimental studies for both FPSOs and semi-submersibles that the wavecurrent interaction effect can be much larger than the effects from current and waves alone.

Direct calculation of wave drift forces in random waves by a Computational Fluid Dynamic tool solving Navier-Stokes equation is still challenging in terms of computational effort. The $26^{\text {th }}$ ITTC (2011) Specialist Committee on CFD indicated that, for seakeeping applications, the present CFD solutions are far too slow in terms of solution time to impact design at an early stage.

Significant progress in terms of accuracy and efficiency has been made on the time-domain fully nonlinear potential flow method in recent years. Among others, Yan \& Liu (2011) used pre-corrected fast Fourier transform (pFFT) algorithm to accelerate their quadratic Boundary Element Method (BEM) for seakeeping analysis. Recent development in volume methods for fully nonlinear wave body interaction studies also suggest that computational time and memory requirement may not be a major concern. For example, a higher order finite difference method was used by Ducrozet et al. (2010) to study nonlinear wave diffraction of a bottom-mounted cylinder. Shao \& Faltinsen (2014a, 2014b) developed an efficient and accurate Harmonic Polynomial Cell (HPC) method and applied their method to various problems in marine hydrodynamics. A fully nonlinear potential flow solver becomes unstable when the wave is breaking, which occurs quite often in reality at the waterline of the structures. Reliable numerical schemes need to be developed to consistently deal with waves before and after breaking.

The leading order nonlinear effects governing the slow-drift response of floating structures depend quadratically on the wave amplitude. In a random wave environment, a complete account of all quadratic wave effects requires the solution of the linear, second- and third-order free surface problems, as discussed in Sclavonous (1992). In typical sea spectra, however, low frequency quadratic effects are dominated by the linear and second-order free surface problem. From this point of view, it may be sufficient to consider the linear and difference-frequency wave-body interaction problems by a second-order nonlinear theory.

In a recent study by Shao (2016) as part of the ExWave Joint Industry Project, fully nonlinear wave diffraction of bottom mounted cylinder in regular waves with different wave steepness was studied to assess the higher than second order effects 
on the mean drift forces. Typical diameters of the columns of semi-submersibles were used in the study. The wave period was based on 1-year storm in the North Sea. The study showed that the higher than second order effects can either increase or decrease the wave drift forces, but only to a small amount of approximately $12 \%$ of the total drift forces in the worst cases. The study also demonstrated the relative importance of viscous effect on the wave drift forces, which is a third order effect. The second order mean drift forces were seen to dominate over both viscous and higher order potential flow effects for the cases studied in the JIP. Generalization of the findings in this study will need further studies, especially for ship-shaped structures.

Among others, early examples of the pioneering work on the study of second order wave-body interaction can be found in Faltinsen \& Løken (1978) and Molin (1979). In a general second-order wave-structure analysis, the convergence of the integral of quadratic terms in the hydrodynamic pressure can be very slow, especially for structures with sharp edges. Middle-field formulation (e.g. Chen 2007, Liang \& Chen 2017) or momentum theory based formulations (e.g. Lee 2007; Xiang \& Faltinsen 2010) have been proposed to avoid this difficulty. Another difficulty associated with second-order wave-structure analysis is the second derivatives in the body boundary conditions, which are not integrable in case of sharp corners and difficult to calculate for structures with large curvature. One example of the second derivatives is the $m$-terms in the seakeeping analysis when the local steady flow effects are considered. Shao \& Faltinsen (2010a, 2010b) proposed a new formulation for the second-order wave-body interaction problem in a body-fixed coordinate system to avoid all the higher order derivatives in the body boundary conditions. It was later extended by Shao \& Faltinsen (2012) for a second-order time-domain wave-current-body interaction model to include a steady current effect. The use of bodyfixed coordinate system also avoids the inconsistency in the traditional perturbation method when the second order slowly vary motions are larger than the linear motions.

In the time domain analysis of wave-structure interaction with the presence of steady or slowly varying velocities, special care has to be taken to stabilize the solution. In this paper, a stabilized numerical method based on streamline integration and biased differencing scheme along the streamlines is applied. The streamline integration method is a popular strategy in finite element analysis (see Zienkiewicz et al., 2014; Servan-Camas, 2016) operating on unstructured meshed to deal with the convective terms. The presence of convective terms in the kinematic and dynamic free surface conditions will lead to unstable solutions if an explicit method is applied. An implicit scheme is used in this paper for the time integration of kinematic and dynamic free surface conditions. In an implicit scheme, solving an additional matrix equation is normally required due to the presence of convective terms. This is associated with the fact that spatial derivatives on the free surface are calculated using the variables at the present time step rather than the previous time steps. Thus, a method that avoids 
solving such matrix equation is developed, which will reduce the computational efforts in the implicit method. The methodology is applicable to both structured and unstructured meshes.

The present study concerns the weakly-nonlinear potential-flow hydrodynamic effects and assumes that the viscous effects can be accounted for empirically. This assumption is often good enough for large volume structures, for which the inertia forces are dominant. As future work, it is also straightforward to include the wave and current forces due to the presence of slender structures, for instance, the inertia and drag forces by Morison equation into the present time-domain model. This is assuming that adequate drag and inertia coefficients can be obtained through dedicated model tests or CFD analysis.

\section{BOUNDARY VALUE PROBLEM IN A BODY-FIXED COORDINATE SYSTEM}

\subsection{Governing equation and coordinate systems}

When the fluid (water) is assumed to be incompressible and inviscid, and the flow irrotational, the water motion can be described by a scalar $\phi$, defined as velocity potential, satisfies the Laplace equation

$$
\nabla^{2} \phi=0 \quad \text { in the fluid domain. }
$$

Since we are going to use a perturbation scheme in the following analysis, the amplitudes of the incident waves assumed asymptotically small. The motions due to steady or slowly varying structure velocities are not assumed as small in the perturbation method that will follow.

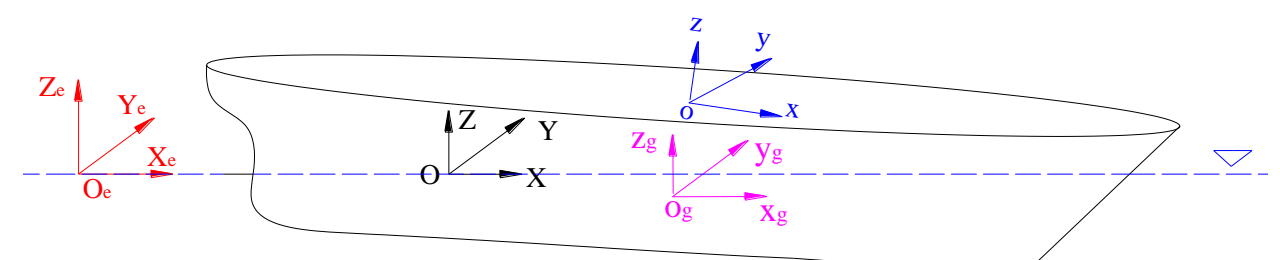

Fig. 1 Definition of different coordinate system.

To facilitate the formulation of boundary conditions in the following sessions, four different right-handed Cartesian coordinate systems, i.e. $O_{e} X_{e} Y_{e} Z_{e}, O X Y Z, o x y z$ and $o_{g} x_{g} y_{g} z_{g}$ are defined. $O_{e} X_{e} Y_{e} Z_{e}$ is Fig. 1 shows an illustration of different coordinate systems. Earth-fixed with $X_{e} Y_{e}$-plane on the calm water surface and the $Z_{e}$-axis positive upwards. $O X Y Z$ is a coordinate system which has the same translatory and rotational velocities as steady or slowly varying velocities of the structure in the horizontal plane. In case of a ship with constant forward speed on a straight course, $O X Y Z$ is an inertial coordinate system. For an offshore platform with slowly varying translatory and rotational velocities in the 
horizontal plan, $O X Y Z$ system is a non-inertial coordinate system. The $X Y$-plane coincides with the $X_{e} Y_{e}$-plane and the $Z$-axis is parallel to the $Z_{e}$-axis. The non-inertial coordinate system oxyz is body-fixed which moves with not only the steady and slowly varying structure velocities but also the wave-frequency unsteady rigid-body motions of the body. When the body does not have wave-frequency unsteady motions, oxyz coincides with $O X Y Z$ with the origin on the mean free surface. The $o_{g} x_{g} y_{g} z_{g}$ coordinate system is an inertial coordinate system with its origin located on the center of gravity, and its axis parallel to that of OXYZ.

In the following formulations of Boundary Value Problems (BVPs) based on a perturbation scheme, the steady or slowlyvarying velocities will assumed as $O(1)$, while the incident wave amplitude and wave-frequency unsteady rigid-body motions of the body are assumed as asymptotically small.

In a traditional perturbation method for linear and weakly nonlinear wave-structure analysis, the body boundary condition is Taylor expanded with respect to the equilibrium position of the structure. It is also assumed that the second order motions are asymptotically smaller than the first order motions. For moored offshore structures, the second order slowdrift motions in horizontal plan is normally larger than their corresponding first order components. In storm seas, they can also be of the same order of magnitude as the characteristic dimensions of the structures.

In this paper, the free-surface and body-boundary conditions are formulated in the oxyz coordinate system, which avoid the inconsistency in the traditional perturbation method in the Earth-fixed coordinate system. The resulting formulation will also avoid evaluation of second derivatives on the body surface.

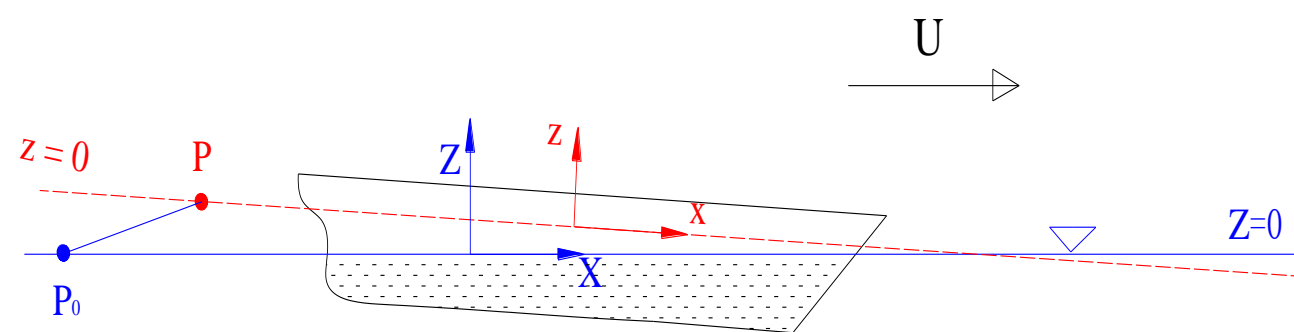

Fig. 2 The relationship between the calm-water surface and the $\boldsymbol{x y}$-plane. 


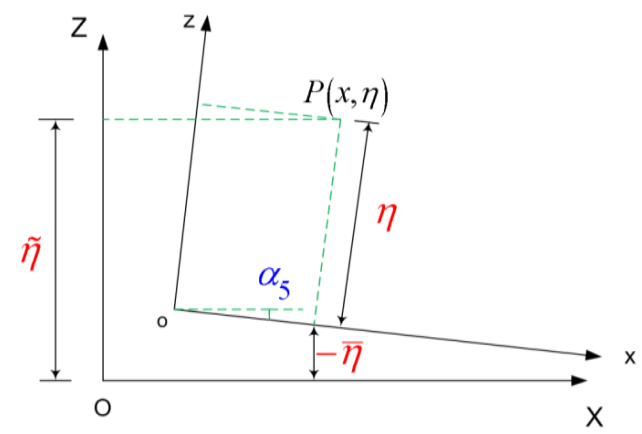

Fig. 3 Definition of the wave elevations observed in the body-fixed coordinate system oxyz and the inertial coordinate system $\boldsymbol{O X Y Z}$.

\subsection{Free surface condition}

The fully-nonlinear formulation of the free-surface conditions in a non-inertial coordinate system can be found in, for instance Faltinsen \& Timokha (2009), and they can written as follows

$\eta_{t}=\phi_{z}-\bar{\nabla} \phi \cdot \bar{\nabla} \eta-\left(\vec{V}^{\prime}+\vec{\Omega}^{\prime} \times \vec{r}^{\prime}\right) \cdot\left(-\eta_{x},-\eta_{y}, 1\right)$ on $z=\eta(x, y, t)$

$\phi_{t}=-\frac{1}{2} \nabla \phi \cdot \nabla \phi+\left(\vec{V}^{\prime}+\vec{\Omega}^{\prime} \times \vec{r}^{\prime}\right) \cdot \nabla \phi-U_{g}$ on $z=\eta(x, y, t)$

Here the subscripts $x, y, z$, and $t$ indicate partial differentiation. $\vec{r}^{\prime}=(x, y, \eta)$ is the position vector of a point on the free surface. $\vec{V}^{\prime}$ and $\vec{\Omega}^{\prime}$ are translatory and rotational body motions, respectively. All the vectors are described in the bodyfixed coordinate system, i.e. oxyz in Fig. 1. The gradients are taken with respect to $x, y$ and $z$, i.e. $\nabla=\vec{\imath} \frac{\partial}{\partial x}+\vec{\jmath} \frac{\partial}{\partial y}+\vec{k} \frac{\partial}{\partial z}$ and $\bar{\nabla}=\vec{\imath} \frac{\partial}{\partial x}+\vec{\jmath} \frac{\partial}{\partial y}$. $U_{g}$ is the gravity potential. For a point $\vec{r}^{\prime}=(x, y, \eta)$ on the free surface, $U_{g}$ can be expressed as

$$
U_{g}=-\vec{g} \cdot\left[\vec{\xi}+R_{b \rightarrow i} \vec{r}^{\prime}\right]
$$

Here $\vec{g}=-g \vec{K} \cdot \vec{\xi}=\xi_{1} \vec{I}+\xi_{2} \vec{J}+\xi_{3} \vec{K}$ is the translatory motion vector of the origin of oxyz relative to $O X Y Z$ system. $\vec{I}$, $\vec{J}$ and $\vec{K}$ are unit vectors along $X$-, $Y$ - and $Z$-axis, respectively. $R_{b \rightarrow i}$ is the transformation matrix that transfers a vector in oxyz coordinate system to its representation in $O X Y Z$ coordinate system.

The free-surface conditions (2) and (3) are then linearized by introducing Stokes expansion and Taylor expanding the free-surface conditions about the $x y$-plane. One should note that the $x y$-plane is not necessarily the same as the calm water surface, i.e. the $X Y$-plane in Fig. 2. The $x y$-plane coincides with the calm water surface when the body is at rest, but translates and rotates with the structure. As shown in Fig. 2, a point $P_{0}$ initially on the calm water surface will move to point $P$ due to unsteady rigid-body motions. Because the perturbation scheme assumes that the wave amplitude and body motions are asymptotically small, if we truncate the computational domain at a finite distance away from the body, 
the distance $\left|P_{0} P\right|$ will be small compared with the characteristic dimensions of the ship (i.e. length, beam and draft). This makes the linearization of the free-surface condition valid within a finite distance to the body.

The free-surface elevation observed in the body-fixed coordinate system has two contributions. The first part is due to the rigid-body motions. It can be understood as follows. When the water is calm, i.e. no incoming or scattered waves, the calm water surface has a relative motion observed in the body-fixed reference frame due to the unsteady body motions. The other contribution is associated with the wave motion with the mentioned rigid-body motion effect excluded. Fig. 3 shows a two-dimensional sketch of the definition of the wave elevation $\tilde{\eta}$ observed in the coordinate system $O X Y Z$ and the wave elevation $\eta$ observed in the body-fixed coordinate system oxyz. $P(x, \eta)$ is a point on the instantaneous free surface. $(-\bar{\eta})$ is the displacement of a point $(x, y, 0)$ on the $x y$-plane due to the rigid-body motions. Keeping in mind that the rigid-body motions and the wave elevation are small, we can approximate the relationship between $\eta$ and $\tilde{\eta}$ as

$$
\eta=\bar{\eta}+\tilde{\eta}+O\left(\epsilon^{3}\right)
$$

From a numerical implementation point of view, it was found advantageous to use the decomposition in Eq.(5) and to operate with $\tilde{\eta}$ instead of $\eta$ in the free-surface conditions.

By introducing the Stokes expansion of the velocity potential $\phi$, wave elevation $\tilde{\eta}$, wave-frequency translational body motion $\vec{\xi}$ and rotational body motion $\vec{\alpha}$, Taylor expanding the free-surface conditions about the $x y$-plane of the bodyfixed coordinate system, and collecting consistent terms at different orders, we can get the following linearized freesurface conditions

$$
\left(\frac{\partial}{\partial t}-\left(\vec{W}^{\prime(0)}-\bar{\nabla} \phi^{(0)}\right) \cdot \bar{\nabla}\right) \phi^{(1)}=-g \tilde{\eta}^{(1)}+\vec{W}^{\prime(0)} \cdot \nabla \phi^{(0)}-\frac{1}{2} \nabla \phi^{(0)} \cdot \nabla \phi^{(0)}+\left[\left(\vec{\chi}^{\prime(1)}+\vec{W}^{\prime(1)}\right) \cdot \nabla \phi^{(0)}\right] \quad \text { on } z=0,
$$

$$
\left(\frac{\partial}{\partial t}-\left(\vec{W}^{(0)}-\bar{\nabla} \phi^{(0)}\right) \cdot \bar{\nabla}\right) \tilde{\eta}^{(1)}=\phi_{z}^{(1)}+\tilde{\eta}^{(1)} \phi_{z z}^{(0)}+\left[\bar{\eta}^{(1)} \phi_{z z}^{(0)}-\bar{\nabla} \phi^{(0)} \cdot \bar{\nabla} \bar{\eta}^{(1)}\right] \quad \text { on } z=0 .
$$

Here $\vec{W}^{\prime(0)}(x, y, t)=(U-\Omega y) \vec{\imath}+(V+\Omega x) \vec{\jmath}+0 \cdot \vec{k}$, where $U, V$ and $\Omega$ are the steady (or slowly varying) surge, sway and yaw velocities of the structure. $\phi^{(0)}$ is the double-body steady velocity potential, which satisfies the rigid-wall condition on $x y$-plane. $\phi^{(1)}$ is the linear unsteady velocity potential. $\vec{\chi}^{\prime(1)}$ is the velocity of a point on $x y$-plane due to rigid-body motion relative to the origin of the $O X Y Z$ system, which is defined as

$$
\vec{\chi}^{\prime(1)}=\overrightarrow{\dot{\xi}}^{(1)}+\overrightarrow{\dot{\alpha}}^{(1)} \times \vec{r}^{\prime}=\left(\dot{\xi}_{1}^{(1)}-y \dot{\alpha}_{6}^{(1)}\right) \vec{\imath}+\left(\dot{\xi}_{2}^{(1)}+x \dot{\alpha}_{6}^{(1)}\right) \vec{\jmath}+\left(\dot{\xi}_{3}^{(1)}+y \dot{\alpha}_{4}^{(1)}-x \dot{\alpha}_{5}^{(1)}\right) \vec{k}
$$


An over dot means time differentiation. $\vec{W}^{\prime(1)}$ is the first-order component of the steady (or slowly varying) velocities observed in the body-fixed coordinate system, defined as

$$
\vec{W}^{\prime(1)}=\boldsymbol{R}_{i \rightarrow b}^{(1)} \vec{W}^{\prime(0)},
$$

where $\boldsymbol{R}_{i \rightarrow b}^{(1)}$ is the first order transformation matrix that transfers a vector in $O X Y Z$ coordinate system to its representation in oxyz coordinate system.

$\bar{\eta}^{(1)}$ in Eq.(8) is the first-order component of $\bar{\eta}$, expressed as

$$
\bar{\eta}^{(1)}=-\dot{\xi}_{3}^{(1)}-y \dot{\xi}_{4}^{(1)}+x \dot{\xi}_{5}^{(1)}
$$

In Eq.(6) and Eq.(7), the terms in the square brackets are additional terms compared with the linear free-surface conditions formulated in $O X Y Z$ coordinate system, which follows the structure with the same steady or slowly varying velocities. They are dependent on the rigid-body unsteady motions. These terms do not introduce additional difficulties from a computational point view, as the highest derivative term involved is $\phi_{z z}^{(0)}$, which also needs to be calculated in a traditional formulation in an inertial coordinate system.

\subsection{Body-boundary condition}

The linearized body-boundary condition in the body-fixed coordinate system oxyz is

$$
\phi_{n}^{(1)}=\vec{n}^{\prime} \cdot\left(\vec{W}^{\prime(1)}+\vec{u}^{\prime(1)}\right), \text { on } S B
$$

Here $\vec{n}^{\prime}$ is the normal vector on the body surface in body-fixed oxyz coordinate system. $\vec{W}^{\prime(1)}$ describes the angle of attack effect from the steady (or slowly varying) body velocities, defined in Eq.(9). $\vec{u}^{\prime(1)}={\overrightarrow{\dot{\xi}^{\prime}}}^{(1)}+\overrightarrow{\dot{\alpha}}^{(1)} \times \vec{r}^{\prime}$ is the first order rigid-body motion vector measured in body-fixed coordinate system. ${\overrightarrow{\dot{\xi}^{\prime}}}^{(1)}$ and $\overrightarrow{\dot{\alpha}}^{\prime(1)}$ are the linear translatory and angular velocity vectors, respectively. $\vec{r}^{\prime}=(x, y, z)$ is a position vector of a point on the body surface.

$S B$ is the body surface at the instantaneous position. The latter fact avoids introduction of e.g. the $m_{j}$-terms. Only the mean wetted part of the body surface is needed in the formulation. The effect of small variation of the wetted body surface due to the wave elevation and body motion will be handled by Taylor expansion about the $x y$-plane of the body-fixed coordinate system. 


\subsection{Forces and moments calculation}

Pressure integration is used to get the hydrodynamic forces and moments acting on the body. The pressure needed is given by the Bernoulli's equation. However, the Bernoulli's equation is a result of integrating the Euler equations, which are only valid in an inertial system, so we can not directly apply it to an accelerated system. The time derivative of velocity potential in Bernoulli's equation should be replaced by

$$
\left.\frac{\partial}{\partial t}\right|_{\text {non-inertial }}=\left.\frac{\partial}{\partial t}\right|_{\text {inertial }}+\vec{v}_{b} \cdot \nabla
$$

where $\vec{v}_{b}$ is the velocity at a point $(x, y, z)$ due to rigid-body motions. $\nabla$ means spatial gradient.

Transforming the time derivative in the inertial coordinate system into that in the body-fixed reference frame, and integrating the pressure on the body surface, we can express the forces and moments in either the inertial coordinate system or the body-fixed coordinate system. For completeness, the expressions for linear and second order mean forces and moments are included in the Appendix.

\section{CUBIC-ORDER BOUNDARY ELEMETN METHOD}

The 12-node cubic higher-order boundary elements (HOBEM) are used to discrete the boundary surfaces enclosing the fluid domain. In this study, the open source program GMSH (Geuzaine \& Remacle, 2009) is used to generate the meshes on both free surface and body surfaces. Example of elements on mean free surface and a bottom mounted vertical cylinder are shown in Fig. 4.

Fig. 4. 12-node cubic order boundary elements on mean free surface and a bottom mounted vertical cylinder.

An example of the element is shown in Fig. 5 with its mapping in $\xi-\eta$ plane in Fig. 6 . The boundary geometry, the velocity potential and its normal derivative are approximated by 


$$
\begin{aligned}
& {[x, y, z]=\sum_{j=1}^{12} N_{j}(\xi, \eta)\left[x_{j}, y_{j}, z_{j}\right]} \\
& \phi^{(1)}=\sum_{j=1}^{12} N_{j}(\xi, \eta)\left(\phi^{(1)}\right)_{j^{\prime}} \\
& \frac{\partial \phi^{(1)}}{\partial n}=\sum_{j=1}^{12} N_{j}(\xi, \eta)\left(\frac{\partial \phi^{(1)}}{\partial n}\right)_{j}
\end{aligned}
$$

Here $\left(\phi^{(1)}\right)_{j}$ and $\left(\frac{\partial \phi^{(1)}}{\partial n}\right)_{j}$ are the velocity potential and its normal derivative at the $j$-th node of the reference element respectively. The superscript $m$ indicates the order of the magnitude. The 12-node elements are also called incomplete cubic order elements as the interior points are not used. $N_{j}(\xi, \eta)$ with $j=1,2, \ldots, 12$ are the cubic shape functions.

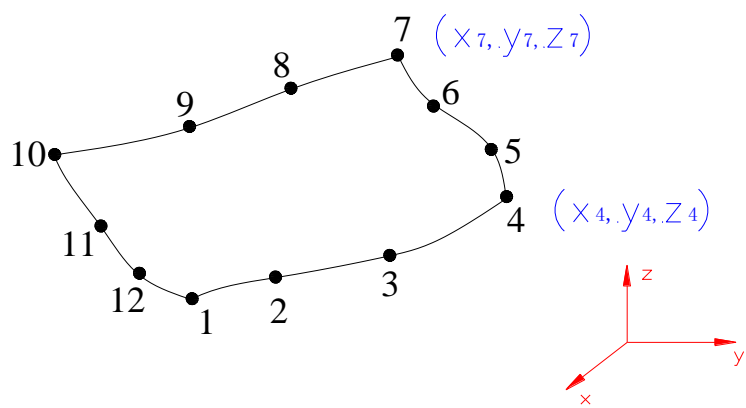

Fig. 5 The 12-node cubic element in the physical plane.

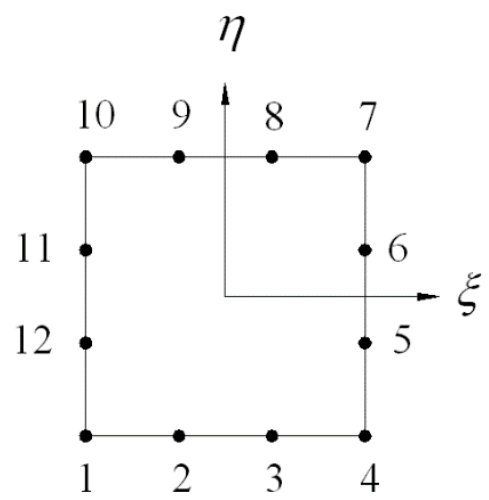

Fig. 6 The 12-node cubic element in the $\boldsymbol{\xi}-\boldsymbol{\eta}$ plane. The numbers are indices for the transformed coordinates $\left(\boldsymbol{\xi}_{\boldsymbol{j}}, \boldsymbol{\eta}_{\boldsymbol{j}}, \mathbf{0}\right)$ corresponding to the coordinates $\left(\boldsymbol{x}_{j}, \boldsymbol{y}_{j}, \boldsymbol{z}_{j}\right)$ in the physical plane. The lengths of the four sides are identical and equal to 2.

The boundary integral equation which is a consequence of applying Green's $3^{\text {rd }}$ theorem to Laplace equation can be solved by the HOBEM. More details on Laplace equation solver based on HOBEM can be found in Shao \& Faltinsen (2014c). 


\section{STABALIZED TIME INTEGRATION OF FREE SURFACE CONDITIONS}

\subsection{Explicit and implicit scheme for time integration}

In the time-domain analysis, the dynamic and kinematic free surface conditions are integrated in time domain from a previous time step to obtain the velocity potential and wave elevation at a new time step. Both the dynamic and kinematic free surface conditions can be written in the following form:

$$
\frac{\partial \psi}{\partial t}+\vec{c} \cdot \bar{\nabla} \psi=f(x, y, t)
$$

Here $\bar{\nabla}=\vec{\imath} \frac{\partial}{\partial x}+\vec{\jmath} \frac{\partial}{\partial y}$ is the differential operator in the horizontal plane. $\psi$ can be understood as either velocity potential or wave elevation on the mean free surface. $f(x, y, t)$ can be understood as the forcing term in the corresponding free surface condition.

If an explicit Euler time integration method is applied, the solution at a new time step $n+1$ can be obtained by using the following discretization

and

$$
\frac{\left\{\psi^{n+1}\right\}-\left\{\psi^{n}\right\}}{\Delta t}+[B]\left\{\psi^{n}\right\}=\left\{f^{n}\right\},
$$

$$
\left\{\psi^{n+1}\right\}=\left\{\psi^{n}\right\}+\Delta t\left\{f^{n}\right\}-\Delta t[B]\left\{\psi^{n}\right\}
$$

Here the superscript indicates the time step number. Matrix [B] is an operator on $x y$ plan to calculate $\vec{c} \cdot \bar{\nabla} \psi$ based on the values of $\psi$ on a given set of free surface points. $\{\psi\}$ is a vector with its elements as $\psi_{i}\left(x=x_{i}, y=y_{i}\right)$ and $j=$ $1,2, \ldots, N$. The stability of the explicit scheme depends how the convective terms are calculated.

Discretizing the equation in both space and time, an implicit scheme takes the following form

$$
\frac{\left\{\psi^{n+1}\right\}-\left\{\psi^{n}\right\}}{\Delta t}+[B]\left\{\psi^{n+1}\right\}=\left\{f^{n}\right\},
$$

which differs from the explicit scheme as the convective terms are approximated by using $\{\psi\}$ at the present time step. The forcing terms represented by $f(x, y, t)$ in Eq.(16) are not explicitly dependent on $\psi$, which can be understood from the free surface conditions in Eq.(6) and (7). For example, the dynamic free surface condition shown in Eq.(6) does not involve $\phi^{(1)}$ and its derivatives in time or space. Strictly speaking, $\phi^{(1)}$ and $\tilde{\eta}^{(1)}$ are coupled through Eq.(6) and (7), thus a complete implicit scheme should evaluate the forcing terms $f(x, y, t)$ at $n+1$ time step. However, the forcing terms are less important than the convective terms when it comes to the stability of the scheme. The presented scheme in (19) is called implicit scheme because of the way the convective terms are handled.

Eq.(19) can be rewritten as 


$$
[A]\left\{\psi^{n+1}\right\}=[[I]+\Delta t[B]]\left\{\psi^{n+1}\right\}=\left(\left\{\psi^{n}\right\}+\Delta t\left\{f^{n}\right\}\right)
$$

Here we have defined $[A]=[I]+\Delta t[B]$. In a seakeeping analysis where both the free surface and body surfaces are discretized with unknowns assigned to the boundary nodes, the free surface normally contains most of the unknown. This indicates that the dimension of matrix $[A]$ is not small. Solving the matrix equation above at each time step will definitely increase the computational efforts.

Considering that $\Delta t$ is a small parameter, we can formally write the solution of $[A]^{-1}$ as a series expansion of $\Delta t$. To this end, our implicit scheme can be rewritten as

$$
\left\{\psi^{n+1}\right\}=\left\{\psi^{n}\right\}+\Delta t\left\{f^{n}\right\}-\Delta t[B]\left\{\psi^{n}\right\}+\left[\Delta t^{2}\left(-[B]\left\{f^{n}\right\}+[B]^{2}\left\{\psi^{n}\right\}\right)+O\left(\Delta t^{3}\right)\right]
$$

The first three terms on the right hand side of Eq.(21) is the same as the that of the explicit scheme. The terms in the square bracket are the additional terms for an implicit scheme, which increase the stability of the scheme. Eq.(21) presents an explicit solution to Eq.(20) accurate up to $O\left(\Delta t^{3}\right)$, which does not involve using an iterative solver.

In Shao \& Faltinsen $(2010,2012)$, an explicit $4^{\text {th }}$ order Runge-Kutta method was used for the time integration of the free surface conditions and the convective were kept on the right hand side of discretized free surface conditions in the similar way as Eq.(18). With a weak low-pass filter on the free surface, the method has been applied for problems with small forward speeds with success. In this paper, the convective terms are treated in an implicit manner, which is expected to be much more stable than that in Shao \& Faltinsen $(2010,2012)$. Only the first order Euler scheme is presented in this paper as an example. However, it is an easy task to extend the present methodology to higher order schemes, which will not be explored further here.

\subsection{Upwind and bias finite difference}

In this paper, the operator [B] that is required in the time integration of free surface conditions will be determined by a streamline integration. At each free surface point, the convective term $\vec{c} \cdot \bar{\nabla} \psi$ can be considered as equivalent to spatial derivative of $\psi$ along the streamline, i.e. $\frac{\partial \psi}{\partial l}=\vec{l} \cdot \nabla \phi$. Here $\vec{l}$ is the unit tangential vector on the streamline. 


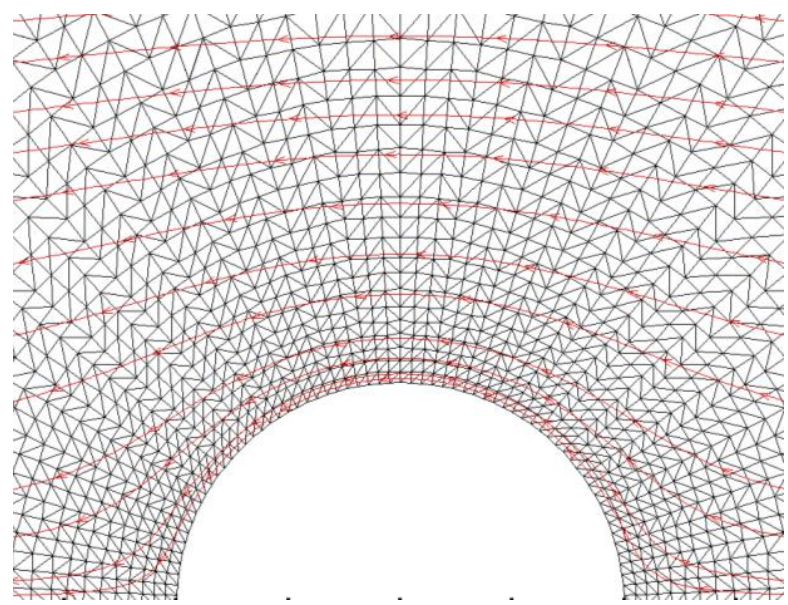

Fig. 7 Streamlines close to a circular cylinder. Only half of the cylinder and streamlines are shown.

Fig. 7 shows some examples of streamlines on the mean free surface close to a vertical circular cylinder. The free surface meshes are also shown in the same figure. To get a fine representation of streamline close to each free surface point, we have sub-divided each 12-node cubic element into 18 triangular elements. The derivative operator for each free surface point is obtained by using a 4-point upwind difference. Fig. 8 shows an illustration of upwind points along a stream line. Since the value of $\psi$ on each of the 4 points is interpolated from the cubic elements which also contain other free surface points, the scheme we applied here is not strictly a pure upwind differencing but a bias finite differencing method for most of the free surface points. A bias finite differencing method is known to have much less numerical dissipation than an upwind scheme with the same number of stencil points. For nodes at the outer boundary of the free surface where the streamline ends, the spatial derivatives are calculated using cubic shape functions on the elements surrounding it.

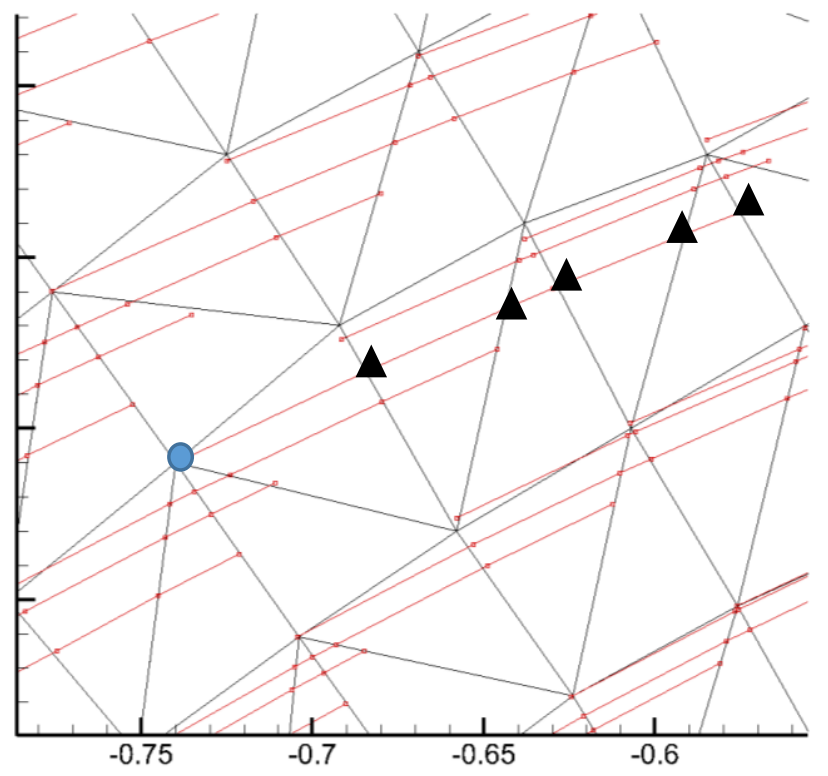

Fig. 8 Upwind points along a streamline. Filled circle is the free point where streamline-differentiation will be performed. Filled triangles are the upstream points next to the point of interest. 


\subsection{Low-pass filters close to the waterline}

When the spatial resolution is too large to represent very short-wave components, one will still expect weak saw-tooth instability. One may overcome it by properly selecting spatial and temporal resolution. In case a fixed spatial resolution is to be applied in different analyses, applying a weak low-pass filter on a few layers of elements close to waterline was seen to be helpful.

One option is to use the following low-pass filter adopted in Shao \& Faltinsen (2014d) on the free surface

$$
\overline{f_{0}}=(1-s) f_{0}+s \sum_{j=1}^{N} w_{j} f_{j}
$$

where $f_{0}$ and $\bar{f}_{0}$ are the values before and after smoothing, respectively. They can be the velocity potential or wave elevation. $s$ is the strength of the low-pass filter. $f_{j}(j=1, \ldots, N)$ are the values on the $\mathrm{j}$-th neighbor node. $w_{j}(j=$ $1, \ldots, N)$ are the weighting coefficients defined as

$$
w_{j}=l_{j}^{2} / \sum_{i=1}^{N} l_{i}^{2}
$$

Here $l_{i}$ is the distance between the point where smoothing will be applied and the $i$-th neighbor point. A closer neighbor point will have larger contribution to the filter. In this study, $s=0 \sim 0.01$ is used, which has negligible damping effect. And it is only applied to about 3-5 layers of elements near the waterline.

The stabilized time integration method presented in this section works not only for small forward problem relevant for offshore engineering but also for ships with larger forward speeds.

\section{VERIFICATION STUDIES}

In order to verify the numerical method described in the previous sections, some numerical tests have been made and are presented in this paper, which show that the proposed numerical method works quite well.

Wave diffraction of a vertical circular cylinder in linear regular waves has been studied as a first case. The cylinder has a draft $d=R$, where $R$ is the radius. The comparison of the amplitude of the linear in-line force with the analytical solution based on MacCamy \& Fuchs's (1954) theory is presented in Fig. 9. The comparison of the present second-order meandrift forces with that of Eatock Taylor \& Hung (1987) is shown in Fig. 10. For the same cylinder, the horizontal mean drift force due to wave diffraction is also presented in Fig. 11 when the cylinder has a small constant forward speed. A Froude number is defined as $F_{r}=U / \sqrt{g R}$ and $F_{r}=-0.01$ is considered in the analysis. Here a negative Froude number means head sea condition. 
The present numerical results agree well with the numerical results of Cheung et al. (1996). Approximately, 8 cubic elements per linear wave length at the waterline are used and a time increment $\Delta t=T / 300$ is adopted in the time-domain simulations. $T$ is the linear wave period of the incident wave.

The horizontal wave drift force on a fixed truncated circular cylinder on truncated vertical circular cylinder has also been studied and the results agree well with the numerical results by Kinoshita \& Bao (1996) and Kinoshita et al. (1997). The cylinder has a draft $\mathrm{h}=2 \mathrm{R}$, with $\mathrm{R}$ as the radius of the cylinder.

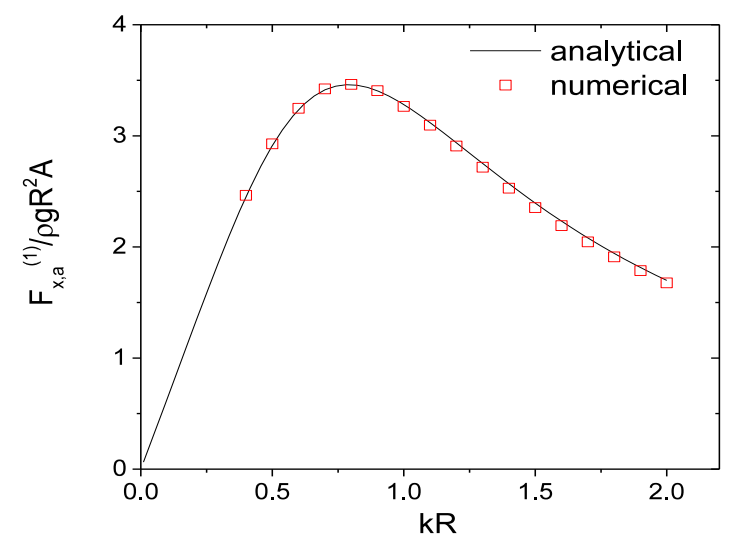

Fig. 9 Comparison of the non-dimensional amplitude of first-order in-line diffraction force with the analytical results based on MacCamy \& Fuchs's (1954) theory. $A$ is the incident wave amplitude. $h=R$.

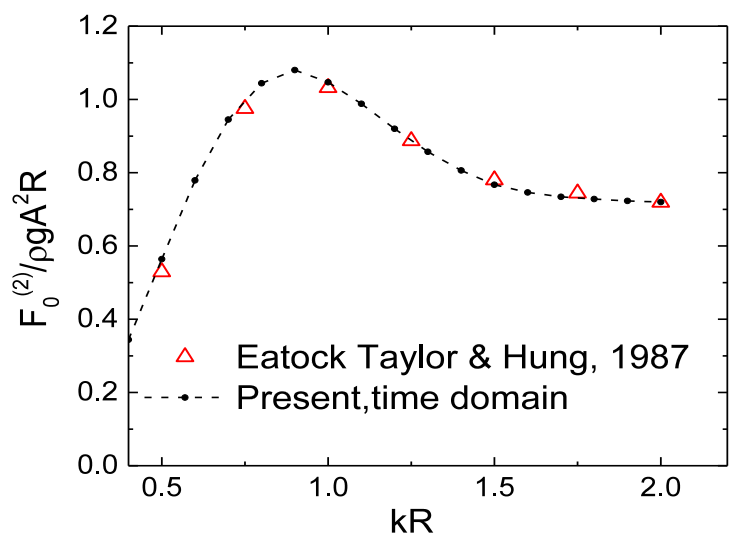

Fig. 10 Comparison of the non-dimensional mean-drift force on a bottom-mounted circular cylinder. $A$ is the incident wave amplitude. $h=R$. 


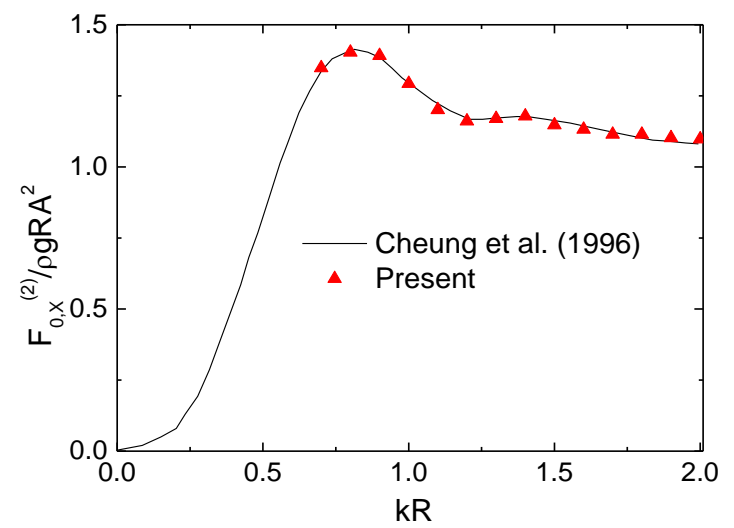

Fig. 11 Non-dimensional horizontal mean drift force on a vertical circular cylinder versus $k R$. $A$ is the wave amplitude. $d=h=R, F r=-0.1 . k$ is the incident wavenumber.

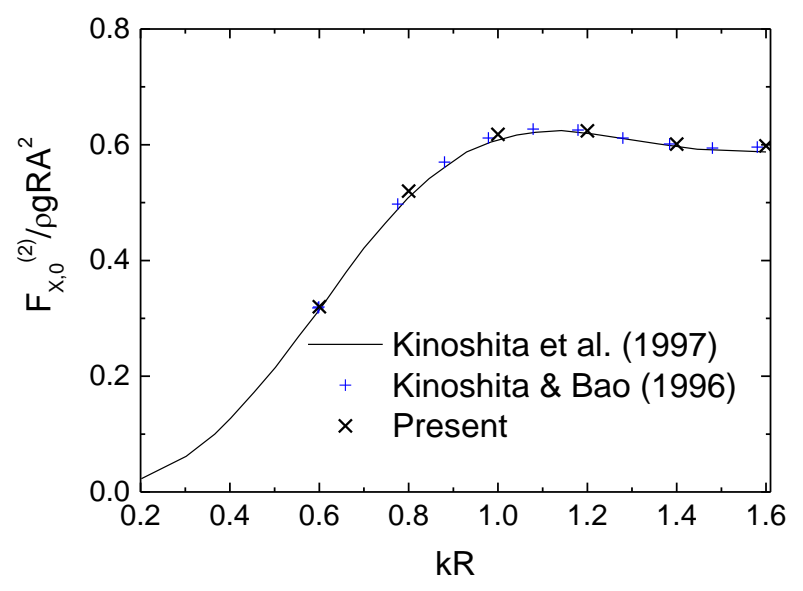

Fig. 12 The horizontal wave drift force on a fixed truncated circular cylinder. $h=2 R, d=R . k$ is the wave number of the incident waves.

Zhao \& Faltinsen (1989b) studied a truncated vertical circular cylinder that is free to oscillate in surge and heave and restrained from oscillating in pitch. A constant panel method (CPM) in the frequency domain was adopted. For zero forward speed case, they obtained the mean wave forces by using both the near-field approach based on direct pressure integration on the body surface and the far-field approach based on momentum relationship. A large discrepancy was found between these methods, which Zhao \& Faltinen (1989b) attributed to the effect of CPM applied to the edge between the bottom and cylinder surface. In theory, the far-field result is much less sensitive to the meshing details on the body surface and is in general more reliable. Liu et al. (1993) computed the same problem employing a frequency-domain HOBEM based on the near-field approach, which resulted in uniform convergence giving a unique result. However, Liu et al.'s (1993) results confirmed neither the far-field result nor the near-field results of Zhao \& Faltinsen (1989b). In fact, 
their results were in between the two results of Zhao \& Faltinsen (1989b). See also the text book of Kim (2008, pp.369$370)$.

The same problem is revisited by using the present method. The force is obtained by the pressure integration on the body surface, i.e. the near-field approach, except that the quadratic force due to the velocity square is re-formulated based on the following equation given in Newman (1977)

$$
\oiint\left[\frac{\partial \phi}{\partial n} \nabla \phi-\frac{1}{2} \nabla \phi \cdot \nabla \phi \vec{n}\right] d s=0
$$

Thus, the integration of the velocity square term in Bernoulli's equation on body surface is rewritten as the summation of two integrals as follows:

$$
\int_{S B} \frac{1}{2} \nabla \phi^{(1)} \cdot \nabla \phi^{(1)} \vec{n} d s=\int_{S B} \frac{\partial \phi^{(1)}}{\partial n} \nabla \phi^{(1)} \vec{n} d s-\int_{S F 1+S C}\left(\frac{\partial \phi^{(1)}}{\partial n} \nabla \phi^{(1)}-\frac{1}{2} \nabla \phi^{(1)} \cdot \nabla \phi^{(1)}\right) \vec{n} d s
$$

Here $S C$ is a control surface enclosed the body surface. $S B$ is the mean body surface. $S F 1$ is a part of the free surface which is confined by the body surface and control surface. This re-formulation improves very much the convergence rate of the quadratic force. The reason is related to the singular behavior of velocity close to the sharp corner. The corner flow solution in the vicinity of the sharp corner can according to Newman (1977) be written as

$$
W(\boldsymbol{Z})=C_{1} \boldsymbol{Z}^{1 / \lambda}+C
$$

where $C_{1}$ and $C$ are constants. $\lambda$ is defined as $\lambda=2-\theta / \pi$ with $\theta$ as the interior angle of the body at the corner. For the a truncated cylinder we have $\theta=\pi / 2$. Therefore, the leading order of the integrand on the left hand side of Eq.(24) is $O\left(r^{-2 / 3}\right)$, while the integrand of the first integral on the right hand side is of $O\left(r^{-1 / 3}\right)$. Here $r$ is the distance of point to the location of the sharp corner. Thus, it is much easer to numerically calculate $\int_{S B} \frac{\partial \phi^{(1)}}{\partial n} \nabla \phi^{(1)} \vec{n} d s$ than $\int_{S B} \frac{1}{2} \nabla \phi^{(1)} \cdot \nabla \phi^{(1)} \vec{n} d s$. The integrands in the second integral on the right hand side of Eq.(24) are not singular.

The considered cylinder has a draft equal to the radius, i.e. $d=R$. The water depth is 1.2 times the linear incident wavelength. As seen in Fig. 13, the time-domain numerical results almost perfectly confirmed the far-field results of Zhao \& Faltinsen (1989b). In the numerical calculations, uniform meshes are used on the cylindrical surface of the cylinder. The elements on the bottom of the cylinder near the corner are of the same size of the elements on the cylinder wall. 
The same problem has also been revisited recently by Mavrakos (2018, personal communication) using a semi-analytical approach (see e.g. Mavrakos 1988) and Liang (2018, personal communication) using both the near-field method and a middle-field formulation implemented in the HydroStar code (see e.g. Chen 2007). Both the semi-analytical results and results based on middle-field formulation agree very well with Zhao \& Faltinsen's (1989b) momentum \& energy conservation based results and the present results. Convergence study has also been made by Liang (2018, personal communication) for the applied near-field method. However, no convergence was achieved even using a very fine mesh. Thus, the near-field results of Liang (2018, personal communication) presented in the Fig.13 are not convergent. They are included only for the purpose to indicate the significant differences between results for an un-convergent near-field method and other methods that are relatively easier to achieve convergence for the vertical mean wave forces on the considered truncated vertical cylinder.

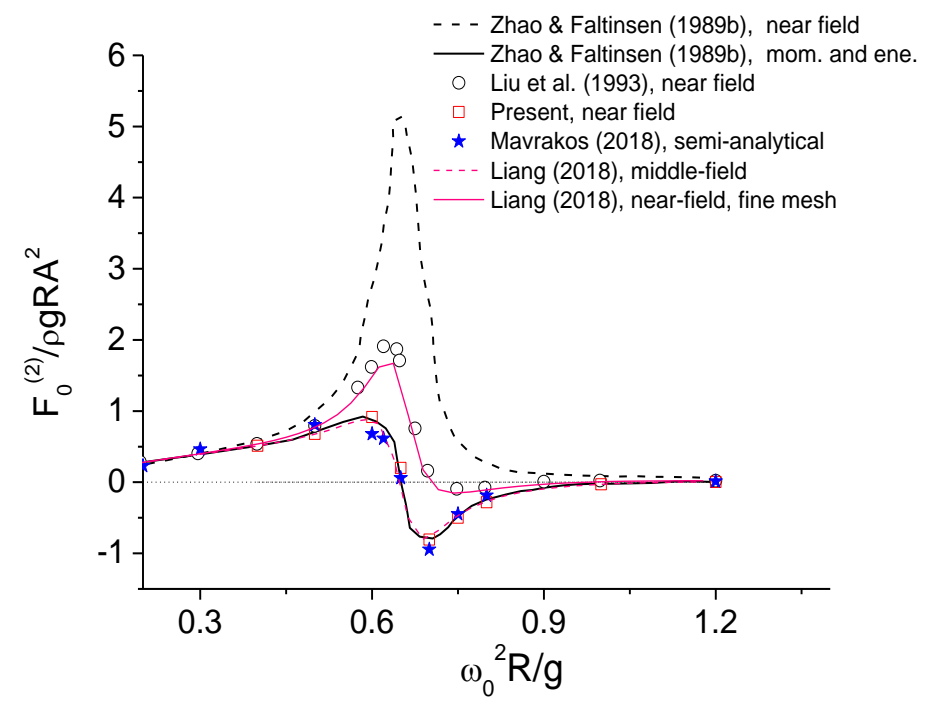

Fig. 13 The numerical results of vertical mean wave force. The quadratic part of the second-order force is calculated based on the re-formulation in Eq.(24). Comparisons with the near-field and far-field results of Zhao \& Faltinsen (1989b) are made. $\boldsymbol{\omega}_{\mathbf{0}}$ is the frequency of the incident wave. $\boldsymbol{R}$ is the radius of the cylinder. $\boldsymbol{g}$ is gravitational acceleration.

\section{SUMMARY AND FUTURE WORK}

A stabilized higher order boundary element method is presented to solve the wave-drift forces with the presence of steady or slowly varying velocities. The method use a formulation of boundary value problem in body-fixed coordinate system and streamline integration. An implicit scheme is used in the time integration of kinematic and dynamic free surface conditions. A method that avoids solving an additional matrix equation which is normally needed in a traditional method has been developed. So far, the method has been verified by mean wave forces on bottom mounted and truncated vertical 
circular cylinder. Future studies will focus on slow-drift motions of marine structures. Wave and current forces on slender elements of the structures may be included through Morison type of equations.

\section{ACKNOWLEDGEMENT}

The author is grateful to Prof. S.A. Mavrakos from and Dr. Hui Liang for carrying out numerical calculations for the vertical mean wave forces on the truncated vertical cylinder presented in the paper. He also would like to thank Prof. S.A. Mavrakos and Prof. B. Molin for interesting discussions on the subject of second order wave loads.

\section{REFERENCES}

[1] Chen, X.B. (2007). Middle-field formulation for the computation of wave-drift loads. J. Eng. Math., 59, 61-82.

[2] Cheung, K.F., Isaacson, M., and Lee, J.W. (1996). Wave diffraction around a three-dimensional body in a current. J. Offshore Mech. Arct. Eng., 118, 4, 247-252.

[3] Ducrozet, G., Bingham H.B., Engsig-Karup, A.P., and Ferrant, P. (2010), High-order finite difference solution for 3D nonlinear wave-structure interaction, Journal of Hydrodynamics, 22(5), supplement: 225-230

[4] Eatock Taylor, R. and Hung S.M. (1987). Second order diffraction forces on a vertical cylinder in regular waves, Appl. Ocean Res., 9, 19-30.

[5] Faltinsen, O.M. and Loken, A.(1978). Drift forces and slowly varying horizontal forces on a ship in waves, Symposium on Applied Mathematics dedicated to the late Prof. Dr. R. Timman. Delft, The Netherlands.

[6] Faltinsen, O.M. and Timokha, A.N. (2009). Sloshing, Cambridge University Press.

[7] Geuzaine, C. and Remacle, J.-F. (2009). Gmsh: a three-dimensional finite element mesh generator with built-in preand post-processing facilities. International Journal for Numerical Methods in Engineering, 79(11), 1309-1331.

[8] Kim, C.H. (2008). Nonlinear waves and offshore structures, World Scientific Publishing.

[9] ITTC (2011). The specialist committee on computational fluid dynamics - final report and recommendation to the $26^{\text {th }}$ ITTC. Available online: https://ittc.info/media/5528/09.pdf.

[10] Kinoshita, T. and Bao, W. (1996). Hydrodynamic forces acting on a circular cylinder oscillating in waves and a small current. J Mar Sci Technol, 1, 155-173.

[11] Kinoshita, T., Bao, W. and Zhu. R. (1997). Higher-order boundary element method for the interaction of a floating body with both waves and slow current. J Mar Sci Technol, 2, 268-279.

[12] Lee, C.H. (2007). On the evaluation of quadratic forces on stationary bodies. J. Eng. Math., 58, 141- 148.

[13] Liang, H. and Chen, X.B. (2017). A new multi-domain method based on an analytical control surface for linear and second-order mean drift wave loads on floating bodies. Journal of Computational Physics. 347, 506-532. 
[14] Liu, Y.H., Kim, C.H. and Kim, M.H. (1993). Computation of mean drift forces and wave run-up by higher-order boundary element method. Int. J. Offshore Polar Eng., 3(2), 101-106.

[15] MacCamy, R. C. and Fuchs, R. A. (1954). Wave forces on piles: a diffraction theory. Report No. TM-69. Corps of Engineers Washington D C Beach Erosion Board.

[16] Mavrakos, S.A. (1988). The vertical drift force and pitch moment on axisymmetric bodies in regular waves. Appl. Ocean Res. 10(4), 207-218.

[17] Molin, B. (1979). Second-order diffraction loads upon three-dimensional bodies, Appl. Ocean Res., 1, $197-202$.

[18] Nestegård, A. and Shao, Y.L. (2014). Wave forces on floating units in extreme waves and current. DNV Report number 2013-1640.

[19] Newman, J. N. (1977). Marine hydrodynamics. Cambridge, MA, MIT Press.

[20] Sclavounos, P.D. (1992), The quadratic effect of random gravity waves on a vertical boundary. J. Fluid Mechs., 242, 475-489.

[21] Servan-Camas, B. (2016), A time-domain finite element method for seakeeping and wave resistance problems. School of Naval Architecture and Ocean Engineering, Technical University of Madrid.

[22] Shao, Y.L. (2010), Numerical potential-flow studies on weakly-nonlinear wave-body interactions with/without small forward speeds, $\mathrm{PhD}$ thesis, Department of Marine Technology, Norwegian University of Science and Technology, Norway.

[23] Shao, Y.L. (2016). Higher order effects on the mean wave drift forces. Project report to ExWave Joint Industry Project.

[24] Shao, Y.L. and Faltinsen, O.M. (2010), Use of body-fixed coordinate system in analysis of weakly nonlinear wavebody problems. Appl. Ocean Res, 32(1), 20-33.

[25] Shao, Y.L. and Faltinsen, O.M. (2012), Second-order diffraction and radiation of a floating body with small forward speed, J. Offshore Mech. Arct. Eng., 135(1).

[26] Shao, Y.L. and Faltinsen, O.M. (2014a), A Harmonic Polynomial Cell (HPC) method for 3D Laplace equation with application in marine Hydrodynamics. Journal of Computational Physics, 274, 312-332.

[27] Shao, Y.L. and Faltinsen O.M. (2014b), Fully-nonlinear wave-current-body interaction analysis by a Harmonic Polynomial Cell (HPC) method, J. Offshore Mech. Arct. Eng., 136(3), 031301-031307.

[28] Shao, Y.L. and Faltinsen, OM (2014c), A numerical study of the second-order wave excitation of ship springing by a higher-order boundary element method, International Journal of Naval Architecture and Ocean Engineering, 4(4), $1000-1013$. 
[29] Shao, Y.L. and Helmers, J.B. (2014d), Numerical analysis of second-order wave loads on large-volume marine structures in a current, 33 ${ }^{\text {rd }}$ International Conference on Ocean, Offshore and Arctic Engineering, July 8-13, 2014, San Francisco, CA.

[30] Stansberg, C.T., Kaasen, K.E., Abrahamsen B.C., Nestegård A., Shao Y.L. and Larsen K. (2015), Challenges in wave force modelling for mooring design in high seas, Offshore Technology Conference, 04-07 May, Houston, Texas, USA.

[31] Xiang, X. and Faltinsen, O.M. (2010). Maneuvering of two interacting ships in calm water. The $11^{\text {th }}$ Practical Design of Ships and Other Floating Structures, Rio de Janeiro, Brazil. Paper no. 2010-2019

[32] Yan, H., and Liu, Y. (2011), An efficient high-order boundary element for nonlinear wave-wave and wave-body interactions, Journal of Computational Physics, 230, 402-424.

[33] Zhao, R. and Faltinsen, O.M. (1989a). A discussion of the mj-terms in the wave-current-body interaction problem. The $3^{\text {rd }}$ Int. Workshop on Water Waves and Floating Bodies.

[34] Zhao, R. and Faltinsen, O.M. (1989b). Interaction between current, waves and marine structures, The $5^{\text {th }}$ Int. Conf. on Num. Ship Hydrodyn. Hiroshima, National Academy Press, Washington D.C.

[35] Zienkiewicz, O.C., Taylor, R.L. and Nithiarasu P. (2014), The finite element method for fluid dynamics, $7^{\text {th }}$ edition, Elsevier Ltd.

\section{APPENDIX}

The linear wave force $\vec{F}^{(1)}$, linear moment $\vec{M}^{(1)}$, the second order mean wave force $\vec{F}_{0}^{(2)}$ and the second order mean wave moment $\vec{M}_{0}^{(2)}$ defined respect to the inertial coordinate system are written respectively as

$$
\begin{aligned}
& \vec{F}^{(1)}=\iint_{S B}\left[p^{(1)} \vec{n}^{(0)}+p^{(0)} \vec{n}^{(1)}\right] d s+\int_{C W_{0}} p^{(0)} \eta^{(1)} \vec{n}^{(0)} / \sqrt{1-n_{3}^{2}} d l \\
& \vec{M}^{(1)}=\iint_{S B}\left[p^{(1)} \vec{m}^{(0)}+p^{(0)} \vec{m}^{(1)}\right] d s+\int_{C W_{0}} p^{(0)} \eta^{(1)} \vec{m}^{(0)} / \sqrt{1-n_{3}^{2}} d l \\
& \vec{F}_{0}^{(2)}=\overline{\iint_{S B}\left[p^{(1)} \vec{n}^{(1)}+p^{(2)} \vec{n}^{(0)}+p^{(0)} \vec{n}^{(2)}\right] d s}+\overline{\iint_{C W_{0}}\left[\frac{1}{2} \rho g\left(\eta^{(1)}\right)^{2} \vec{n}^{(0)}+p^{(0)} \eta^{(1)} \vec{n}^{(1)}\right] / \sqrt{1-n_{3}^{2}} d l} \\
& \vec{M}_{0}^{(2)}=\overline{\iint_{S B}\left[p^{(1)} \vec{m}^{(1)}+p^{(2)} \vec{m}^{(0)}+p^{(0)} \vec{m}^{(2)}\right] d s}+\overline{\iint_{C W_{0}}\left[\frac{1}{2} \rho g\left(\eta^{(1)}\right)^{2} \vec{m}^{(0)}+p^{(0)} \eta^{(1)} \vec{m}^{(1)}\right] / \sqrt{1-n_{3}^{2}} d l}
\end{aligned}
$$


Here $\mathrm{CW}_{0}$ is the mean waterline. $\vec{n}^{(0)}=\left(n_{1}, n_{2}, n_{3}\right)$ and $\vec{n}^{(k)}=\mathbf{R}_{b \rightarrow i}^{(k)} \vec{n}^{(0)}(k=1,2)$ are the components of the normal vector on the body of different orders. Matrix $\mathbf{R}_{b \rightarrow i}^{(k)}$ is the $k$-th order component of the transformation matrix $\mathbf{R}_{b \rightarrow i}$, which transforms a vector in the body-fixed coordinate into its representation in an inertial coordinate system. ' $b$ ' and ' $i$ ' in the subscript mean body-fixed frame and inertial coordinate system, respectively. $\vec{m}^{(0)}=\overrightarrow{\mathrm{r}} \times \overrightarrow{\mathrm{n}}^{(0)}$ and $\vec{m}^{(k)}=\mathbf{R}_{b \rightarrow i}^{(k)} \vec{m}^{(0)}$ are the generalized normal vectors of different orders. $\vec{r}$ is the position vector of a point on the body surface relative to a center, which the moments are defined with respect to. $p^{(k)}$ is the $i$-th order pressure defined respectively as

$$
\begin{gathered}
p^{(0)}=-\rho\left\{g z-\vec{W}^{\prime(0)} \cdot \nabla \phi^{(0)}+\frac{1}{2} \nabla \phi^{(0)} \cdot \nabla \phi^{(0)}\right\} \\
p^{(1)}=-\rho\left\{\phi_{t}^{(1)}-\vec{W}^{\prime(0)} \cdot \nabla \phi^{(1)}-\left(\vec{u}^{\prime(1)}+\vec{W}^{\prime(1)}-\nabla \phi^{(1)}\right) \cdot \nabla \phi^{(0)}+g\left(\xi_{3}^{(1)}+y \alpha_{4}^{(1)}-x \alpha_{5}^{(1)}\right)\right\} \\
p^{(2)}=-\rho\left\{\phi_{t}^{(2)}-\vec{W}^{\prime(0)} \cdot \nabla \phi^{(2)}-\left(\vec{u}^{\prime(1)}+\vec{W}^{\prime(1)}\right) \cdot \nabla \phi^{(1)}-\left(\vec{u}^{\prime(2)}+\vec{W}^{\prime(2)}-\nabla \phi^{(2)}\right) \cdot \nabla \phi^{(2)}+\frac{1}{2} \nabla \phi^{(1)} \cdot \nabla \phi^{(1)}+\right.
\end{gathered}
$$$$
\left.g x_{3}^{(2)}\right\}
$$

$\vec{W}^{\prime(k)}=\mathbf{R}_{i \rightarrow b}^{(k)} \vec{W}^{\prime(0)}$ is the $k$-order component of the steady (or slowly varying) velocities observed in the body-fixed coordinate system. Matrix $\boldsymbol{R}_{i \rightarrow b}^{(k)}$ is the transpose of $\mathbf{R}_{b \rightarrow i}^{(k)} . \vec{W}^{\prime(1)}$ and $\vec{u}^{\prime(1)}$ are already defined in section 2.3 associated with the definition of the body-boundary conditions. $x_{3}^{(2)}$ is the vertical component of the second order displacement at a point on body due to unsteady rigid-body motions. A overbar in Eq.(A.3) and (A.4) means that time average has been taken. 